\title{
Experimental research on optimization of compression molding process parameters of pineapple rind residue
}

\author{
Kunpeng Tian ${ }^{1}$, Bin Zhang ${ }^{1,2}$, Jicheng Huang ${ }^{1,3}$, Haolu Liu ${ }^{1}$, \\ Cheng Shen ${ }^{1,2,4^{*}}$, Xianwang $\mathrm{Li}^{1}$, Qiaomin Chen ${ }^{1}$ \\ (1. Nanjing Institute of Agricultural Mechanization, Ministry of Agriculture and Rural Affairs, Nanjing 210014, China; \\ 2. School of Mechanical Engineering, Southeast University, Nanjing 211189, China; \\ 3. College of Mechanical and Electrical Engineering, Hohai University, Changzhou 213022, Jiangsu, China; \\ 4. Key Laboratory of Modern Agricultural Equipment and Technology, Ministry of Education, \\ Zhenjiang 212013, Jiangsu, China)
}

\begin{abstract}
Currently, the process parameters for compression molding of pineapple rind residue are not clear. In view of this problem, a single die hole compression molding test device was designed in this study, and the force of material in a mold hole was analyzed. Using the test device, a three-factor three-level orthogonal test was carried out by using the particle size, moisture content, and die hole length-to-diameter ratio of pineapple rind residue as the factors and the particle molding rate, relax density, and specific energy consumption as the indicators. The test results were analyzed by range analysis, variance analysis, and fuzzy comprehensive evaluation. The test results show that the main and secondary factors affecting the comprehensive performance of pineapple rind residue compression molding are length-to-diameter ratio, particle size, and moisture content. The optimal parameter combination is the material particle size of $6-9 \mathrm{~mm}$, moisture content of $16 \%$, and length-to-diameter ratio of $4: 1$. The best indicators under these conditions are particle molding rate of $97.80 \%$, relax density of $1.32 \mathrm{~g} / \mathrm{cm}$, and specific energy consumption of $44.17 \mathrm{~J} / \mathrm{g}$. These research results can provide a reference for the selection of processing parameters and the design of molding equipment.
\end{abstract}

Keywords: pineapple rind residue, compression molding, waste utilization, pellet forming, orthogonal test, process parameter optimization

DOI: $10.25165 /$ j.ijabe.20211403.6041

Citation: Tian K P, Zhang B, Huang J C, Liu H L, Shen C, Li X W, Chen Q M. Experimental research on optimization of compression molding process parameters of pineapple rind residue. Int J Agric \& Biol Eng, 2021; 14(3): 221-227.

\section{Introduction}

Pineapple is a tropical fruit with huge global demand. According to statistics, at present, pineapple planting area accounts for about $35 \%$ of the total tropical fruit planting area in the world $^{[1-3]}$. By 2017, the global pineapple output reached 25.9 million t. Due to the thick rind of pineapple, large amounts of rind residue byproducts are produced during processing, and most of these byproducts are thrown away as garbage. Studies have shown that the byproducts of pineapple rind residue still contain rich beneficial components such as pectin ${ }^{[4]}$, flavonoids ${ }^{[5]}$, phenols ${ }^{[6]}$, and dietary fiber ${ }^{[7]}$. Hence, throwing away the pineapple rind not only causes serious waste of resources, but also

Received date: 2020-07-26 Revision date: 2021-03-23

Biographies: Kunpeng Tian, Assistant Professor, research interests: agricultural mechanization engineering, Email: tiankp2005@163.com; Bin Zhang, Professor, research interests: agricultural engineering, Email: xtsset@hotmail.com; Jicheng Huang, Assistant Professor, research interests: agricultural mechanization engineering, Email: huangjicheng@caas.cn; Haolu Liu, Research Assistant, research interests: agricultural engineering, Email: liuhaolu@caas.cn; Xianwang Li, Professor, research interests: agricultural engineering, Email: xw3871@163.com; Qiaomin Chen, Professor, research interests: agricultural engineering, Email: nnncqm@163.com.

*Corresponding author: Cheng Shen, PhD, Assistant Professor, research interests: agricultural mechanization engineering, Nanjing Institute of Agricultural Mechanization, Ministry of Agriculture and Rural Affairs, No.100 Liuying, Xuanwu District, Nanjing 210014, China. Tel: +86-25-84346078, Email: shencheng@caas.cn. exerts great pressure on the environment. The situation of the comprehensive development and utilization of pineapple residue is quite severe, and the comprehensive development and utilization of pineapple rind residue are also limited ${ }^{[8]}$. Therefore, how to turn waste into wealth and make full use of pineapple rind resources is an important problem for pineapple processing industry to reduce cost, improve economic benefits, and reduce environmental pressure $^{[9]}$. As pineapple rind residue has high moisture content and degrades easily, it is not suitable for long-term storage. The loose, disordered, and differently shaped rind residues are dried in air to reduce moisture and compressed into particles of a fixed shape, thus benefiting long-distance transportation and storage and creating conditions for the subsequent extraction of beneficial ingredients ${ }^{[10]}$. At present, two main types of equipment are available for compressing and forming biomass materials: flat mode and ring mode ${ }^{[11]}$. The working principle of the equipment involves using the squeezing force of die roller to solidify the material. However, because of the diversity of biomass, the characteristics of materials are diverse, and the process conditions required for compression molding are also different. To ascertain optimal process conditions and forming mechanism of different types of biomass pellets, a series of studies have been carried out.

Thoreson et al. ${ }^{[12]}$ studied the effect of molding pressure, moisture content, and particle size on the density of corn straw compression molding. Their results show that moisture content has the most significant effect on molding quality. When the moisture content was $13 \%$, the granulation density reached 
$368 \mathrm{~kg} / \mathrm{m}^{3}$, and when the moisture content was more than $25 \%$, the granulation density clearly decreased, making it unsuitable for granulation. Garcia-Maraver et al. ${ }^{[13]}$ carried out an experimental study on the technological parameters of pressing biomass pellet fuel with olive tree pruning branches and leaves residue. They found that the best granulation conditions were obtained when the moisture content of material residues was $9 \%$, the diameter of die holes was $20-24 \mathrm{~mm}$, and the molding temperature was $40^{\circ} \mathrm{C}$. Yumak et al. ${ }^{[14]}$ conducted a particle compression test on tumbleweed using a hydraulic press and studied the effect of moisture content and hydraulic pressure on molding quality. When moisture content was $7 \%-10 \%$ and molding pressure was 31.4 MPa, the molding effect is the best. Kaewwinud et al. ${ }^{[15]}$ studied the effect of moisture content and particle size of cassava stalk residue on the durability of molded granules. They found that when the particle size of cassava stalk residue was $1.7 \mathrm{~mm}$ and the moisture content was $10 \%$, the durability of manufactured granules is the highest. Shaw et al. ${ }^{[16]}$ reported the characteristics of powdery materials and found that the length-to-diameter ratio of the annular mold hole significantly affects the axial pressure in the mold hole and the friction between mold wall and material, respectively.

Hu et al. ${ }^{[17]}$ conducted cold compression molding experiments on wheat straw under different conditions such as different moisture contents, compression speeds, and die length-to-diameter ratios. Their results showed that when the compression speed was $40 \mathrm{~mm} / \mathrm{min}$, a higher maximum compression density and lower specific energy consumption can be obtained. When the moisture content was $16 \%$ and the length-to-diameter ratio was 5.2, the molding effect is the best. Dong et al. ${ }^{[18]}$ reported that the moisture content plays an important role in the molding of sawdust particles. They carried out compression tests on sawdust particles with a hydraulic press, and found that the optimal moisture content range of sawdust particle molding was $15 \%-18 \%$. Zhang et al. ${ }^{[19]}$ conducted an experimental study on the compact molding of fuel water hyacinth particles. The optimal parameters of water hyacinth pellet fuel conditions were as follows: the pressure of $6 \mathrm{kN}$, temperature of $100^{\circ} \mathrm{C}$, moisture content of $12 \%$, and particle size of $0.58 \mathrm{~mm}$. Under these conditions of process parameters, grain density and radial stress of water hyacinth pellet fuel could reach $1,362.21 \mathrm{~kg} / \mathrm{m}^{3}$ and $1.44 \mathrm{kN}$, respectively. Huang et al. ${ }^{[20]}$ conducted a simulation test and found that the optimal pelleting parameter combination of alfalfa grass was $5.47 \mathrm{kN}$ of extrusion force, $16.96 \%$ of moisture content, and $6.34 \mathrm{~mm}$ of grass particle size. Under this technological parameter combination, the pelleting density was $1.241 \mathrm{~g} / \mathrm{cm}^{3}$.

To sum up, studies on pellet forming mainly focused on sawdust, straw, grass powder, and other materials, while studies on fruit processing byproducts such as pineapple rind residue are rare. Since pineapple rind residue and other fruit materials have high moisture content and high viscosity, they are quite different from ordinary biomass fuel and pellet feed materials, and the corresponding molding process parameters are also quite different. To ascertain the best pellet molding process parameters of pineapple rind residue, a self-developed single die hole compression molding test device was used in this study, taking the pineapple rind residue material particle size, moisture content, and die hole length-to-diameter ratio as the test factors. The particle molding rate, relax density, and specific energy consumption were used as the test indicators to conduct the pineapple rind residue molding test. Fuzzy evaluation method was used to comprehensively evaluate and analyze the test results to determine the optimal parameter combination. The results of this study provide a reference for the selection of pineapple rind residue molding technology parameters and the development of molding equipment.

\section{Design of test equipment}

\subsection{Structural design}

To complete the compression molding test of pineapple rind particles, a single-mode hole compression test device was developed, as shown in Figure 1. The device mainly consists of a base, charging barrel, compression bar, and molding die. The charging barrel is connected with the base by threads, the molding die is buckled in the hole at the upper end of base and pre-tightened with the lower end of charging barrel. The compression bar is matched with the inner wall of the charging barrel, and the material is pushed into the die hole by the compression bar.

Referring to the structural parameters of the existing biomass compression molding mold, the inner diameter of molding mold was taken as $8 \mathrm{~mm}$. To analyze the effect of length-to-diameter ratio of different mold holes on forming indexes, three groups of molding molds with lengths of $32 \mathrm{~mm}, 40 \mathrm{~mm}$, and $48 \mathrm{~mm}$ were designed. Namely, the length-to-diameter ratio of mold holes was $4: 1,5: 1$, and $6: 1$, respectively. The inner diameter and length of the barrel were $20 \mathrm{~mm}$ and $100 \mathrm{~mm}$, respectively.

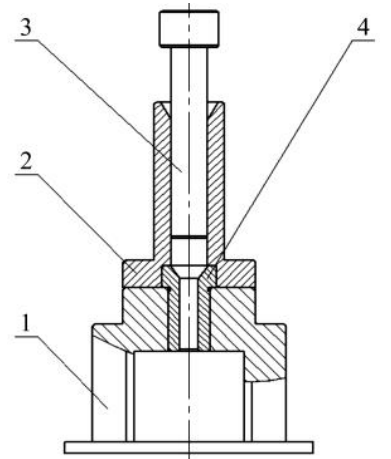

1. Pedestal 2. Charging barrel 3. Compressive bar 4. Forming mold Figure 1 Schematic diagram of single-mode hole compression test device

\subsection{Force analysis of material}

In the particle forming equipment, the cross-sectional area and cross-sectional perimeter of die hole are constant, and the pressure in die hole gradually decreases to atmospheric pressure from the entrance to the exit of granulation die. Under this pressure difference, the materials in the die hole move out of the hole against the friction of side wall. The force analysis of material in the mold hole is shown in Figure $2^{[21]}$.

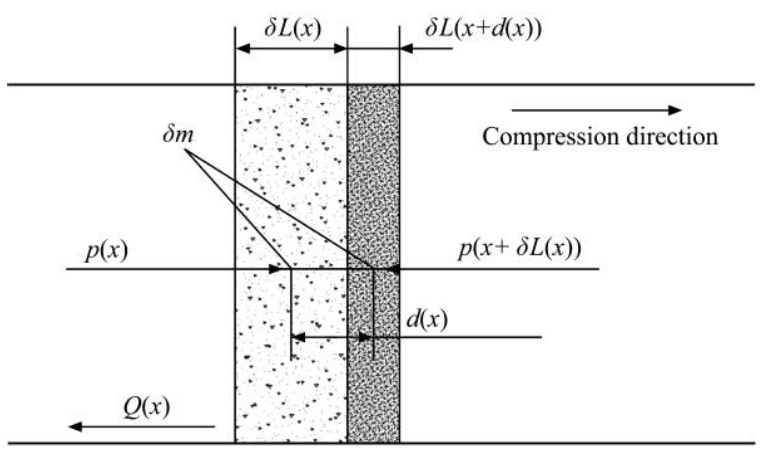

Figure 2 Force analysis diagram of material infinitesimal element in mold hole 
The infinitesimal element taken from the die hole is shown in Figure 2. Its length is $\delta L(x)$, and its mass is $\delta m$. After time $\mathrm{d} t$, its mass $\delta m$ is unchanged, its centroid advancing distance is $d x$, and its length becomes $\delta L(x+\mathrm{d} x)$. The external force acting on the infinitesimal element of material includes gravity (negligible), the pressure of material, and the frictional force of hole wall, where $Q(x)$ is the frictional force, $Q(x)=Z \delta L(x) p(x) f$. The material infinitesimal element produces elastic and plastic deformation when moving, and complex forces exist between the particles in an infinitesimal element. When the infinitesimal element is taken as the research object, the momentum theorem can be used to establish the impulse relationship between the internal and external forces on infinitesimal elements in the corresponding time period, without considering the internal deformation and force of infinitesimal elements.

From the momentum theorem,

$$
\left\{\begin{array}{l}
-[p(x+\delta L(x))-p(x)] S \\
-Z \delta L(x) p(x) f
\end{array}\right\} \mathrm{d} t=\delta m \mathrm{~d} v
$$

where, $p(x+\delta L(x))$ is the pressure (MPa) at point $x+\delta L(x)$, hindering the advance of infinitesimal element with a negative direction; $f$ is the friction factor between the infinitesimal element and die hole wall; $v$ is the advancing speed of centroid of material element; $p(x)$ is the pressure (MPa) in the material at point $x$, which pushes the infinitesimal element forward and the direction is positive; $S$ is the cross-sectional area of die hole, $\mathrm{m}^{2} ; Z$ is the cross-sectional perimeter of die hole, $\mathrm{m}$.

Assuming that the pressure at the outlet of die hole $(x=L)$ is atmospheric pressure $p_{L}$ and the pressure at the inlet of die hole $(x=0)$ is $p_{o}$. It can be got by solving Equation (1),

$$
\frac{S}{Z L}=\frac{f}{\ln \left(\frac{p_{o}}{p_{L}}\right)}
$$

Equation (2) shows that the extrusion force in material compression molding is related to the structural parameters of die hole (cross-sectional area, perimeter, and length of die hole) and the friction coefficient between material and hole wall. Here, the change in structural parameters can be characterized by the length-to-diameter ratio, and the friction coefficient is affected by factors such as material granularity and moisture content. This is also the reason why this study takes the length-to-diameter ratio of die hole, particle size, and moisture content of material as experimental factors to conduct experimental research on material compression molding.

\section{Test materials and methods}

\subsection{Test material}

The test material was pineapple rind residue produced during the processing of canned pineapple in Linyi Lewanjia Food Co., Ltd. (in Linyi, Shandong, China). As the research on the molding of pineapple rind particles is still in the early stage, there is no relevant research report in this field. In view of this, this study drew lessons from the selection principles of material factors given in parameter optimization reports on alfalfa grass ${ }^{[20]}$ and rice straw $^{[22]}$ pellet forming process, and selected moisture content and particle size as the influencing factors of pineapple peel residue material characteristics on pellet forming quality. The material processing method is as follows: pineapple peel residue is naturally dried and crushed, and then screened into three groups of peel residue samples with particle sizes of 0-3 $\mathrm{mm}, 3-6 \mathrm{~mm}$ and 6-9 $\mathrm{mm}$, respectively. According to the three-factor and three-level orthogonal test scheme, the pineapple rind residue of corresponding particle size was dried to the corresponding moisture content, and the moisture content was taken as $(8 \pm 0.5) \%,(16 \pm 0.5) \%$, and $(24 \pm 0.5) \%$.

\subsection{Test equipment}

The instruments and equipment used in the test mainly include a DWD-10 microcomputer controlled electronic universal testing machine (test power range is $10 \mathrm{kN}$, accuracy level is 0.5 ), single mode hole compression test device, SHIMADZU MOC-63U moisture measuring instrument (accuracy is $0.01 \%$ ), BSM-2200 electronic balance (range is $2200 \mathrm{~g}$, accuracy is $\pm 10 \mathrm{mg}$ ), LICHEN-101 drying oven (temperature control range is $10^{\circ} \mathrm{C}$ $250^{\circ} \mathrm{C}$ ), and vernier caliper.

\subsection{Test method}

The experimental design was carried out according to the $\mathrm{L}_{9}\left(3^{4}\right)$ orthogonal table. There were nine groups in total, and each group was repeated three times. The average value was taken. The test factors and level table are shown in Table 1.

Table 1 Orthogonal test factors and levels

\begin{tabular}{cccc}
\hline & \multicolumn{3}{c}{ Factors } \\
\cline { 2 - 4 } Level & $\begin{array}{c}\text { Particle size } \\
A / \mathrm{mm}\end{array}$ & $\begin{array}{c}\text { Moisture content } \\
B / \%\end{array}$ & $\begin{array}{c}\text { Length-to-diameter } \\
\text { ratio } C\end{array}$ \\
\hline 1 & $0-3$ & 8 & $4: 1$ \\
2 & $3-6$ & 16 & $5: 1$ \\
3 & $6-9$ & 24 & $6: 1$ \\
\hline
\end{tabular}

Before the start of the test, the residue was fed into the feed barrel and pressed with the press rod. Then, the single-mode hole compression test device was placed in the loading area of universal testing machine, and the loading speed was set as $40 \mathrm{~mm} / \mathrm{min}$. Then, the universal testing machine was started, and the pressure bar was loaded. The material was extruded into the die hole under the action of press rod and solidified under the action of high pressure due to the change in physical and chemical properties. With the continuous filling of material, the solidified particles were constantly squeezed into the die hole. The extrusion pressure and displacement data were recorded using the universal testing machine. The compression test of cinder material is shown in Figure 3.

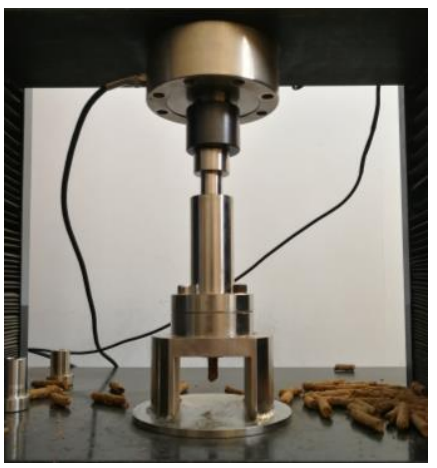

Figure 3 Compression test of cinder material

\subsection{Test indexes}

According to SC/T $6012-2002^{[23]}$, and $\mathrm{NY} / \mathrm{T} 1881^{[24]}$, the granule molding rate, relax density, and specific energy consumption were used as the evaluation indexes.

(1) Molding rate

Procedure: About $200 \mathrm{~g}$ of rind residue particles sample was taken. The particles were sieved completely using a square sieve with a side length to particle diameter ratio of 0.8 , and then 
weighed. To avoid error, the procedure was repeated three times, and the average value was taken. The molding rate can be calculated as follows:

$$
\lambda=\frac{m_{1}}{m} \times 100 \%
$$

Where, $\lambda$ is the molding rate, $\% ; m_{1}$ is the weight of formed particles, $\mathrm{g} ; m$ is the weight of sampled particles, $\mathrm{g}$.

(2) Relax density

The length, diameter, and mass of formed particles were measured using an electronic balance and vernier caliper after placing in a sealed bag for $2 \mathrm{~h}$, and the relax density was calculated from its mass and volume. Relax density can be calculated as follows:

$$
\rho=\frac{4 m}{\pi d^{2} h}
$$

where, $\rho$ is the relax density, $\mathrm{g} / \mathrm{cm}^{3} ; m$ is the mass of sampled particles, $\mathrm{g} ; d$ is the particle diameter, $\mathrm{cm} ; h$ is the particle length, $\mathrm{cm}$.

\section{(3) Specific energy consumption}

According to the pressure, displacement, and particle mass in compression molding, the specific energy consumption can be calculated as follows:

$$
w=\frac{\sum_{i=1}^{n} F_{i} \cdot \Delta x}{m}
$$

where, $w$ is specific energy consumption, $\mathrm{J} / \mathrm{g} ; F_{i}$ is the compression force corresponding to the data collection point $i, \mathrm{~N} ; \Delta x$ is the loading displacement between two adjacent data collection points of universal testing machine, $\mathrm{mm}$.

\subsection{Results and analysis}

3.5.1 Effect of various factors on compression molding performance index

The orthogonal test scheme and test results are shown in Table 2. To determine the effect of each test factor on the index, range analysis and variance analysis were performed on each test index. The results of range analysis and variance analysis are shown in Tables 3 and 4, respectively.

The larger the range was, the greater the influence of change in the test factor level on the test index. Table 3 shows that the primary and secondary order of influence of each factor on the molding rate and relax density is length-to-diameter ratio $C$, particle size $A$, and moisture content $B$, and the optimal parameter combination scheme is $C_{3} A_{1} B_{2}$. The primary and secondary order of influence of each factor on specific energy consumption is length-to-diameter ratio $C$, moisture content $B$, and material particle size $A$, and the optimal combination scheme is $C_{1} B_{1} A_{3}$.

Table 2 Orthogonal test scheme and test results

\begin{tabular}{cccccccc}
\hline No. & $A$ & $B$ & $C$ & Error & $\begin{array}{c}\text { Forming } \\
\text { rate } \lambda / \%\end{array}$ & $\begin{array}{c}\text { Relax density } \\
\rho / \mathrm{g} \cdot \mathrm{cm}^{-3}\end{array}$ & $\begin{array}{c}\text { Specific energy } \\
w / \mathrm{J} \cdot \mathrm{g}\end{array}$ \\
\hline 1 & 1 & 1 & 1 & 1 & 89.49 & 1.08 & 45.83 \\
2 & 1 & 2 & 2 & 2 & 95.52 & 1.24 & 55.07 \\
3 & 1 & 3 & 3 & 3 & 97.20 & 1.31 & 56.51 \\
4 & 2 & 1 & 2 & 3 & 88.75 & 1.13 & 53.02 \\
5 & 2 & 2 & 3 & 1 & 96.19 & 1.29 & 58.57 \\
6 & 2 & 3 & 1 & 2 & 87.12 & 1.07 & 48.29 \\
7 & 3 & 1 & 3 & 2 & 90.50 & 1.14 & 54.46 \\
8 & 3 & 2 & 1 & 3 & 87.14 & 1.00 & 47.88 \\
9 & 3 & 3 & 2 & 1 & 88.82 & 1.10 & 51.99 \\
\hline
\end{tabular}

Note: $A$ is Particle size, $B$ is Moisture content and $C$ is Length-to-diameter ratio,

\begin{tabular}{|c|c|c|c|c|}
\hline \multicolumn{2}{|c|}{ Test index } & $A$ & $B$ & $C$ \\
\hline \multirow{6}{*}{$\begin{array}{c}\text { Forming } \\
\text { Rate } \\
\lambda\end{array}$} & $K_{1}$ & 282.21 & 268.74 & 263.75 \\
\hline & $K_{2}$ & 272.06 & 278.85 & 273.09 \\
\hline & $K_{3}$ & 266.46 & 273.14 & 283.89 \\
\hline & $R$ & 15.75 & 10.11 & 20.14 \\
\hline & \multicolumn{4}{|c|}{ Sequence of factors $C>A>B$} \\
\hline & \multicolumn{4}{|c|}{ Optimal decision $C_{3} A_{1} B_{2}$} \\
\hline \multirow{6}{*}{$\begin{array}{c}\text { Relax density } \\
\rho\end{array}$} & $K_{1}$ & 3.63 & 3.35 & 3.15 \\
\hline & $K_{2}$ & 3.49 & 3.53 & 3.47 \\
\hline & $K_{3}$ & 3.24 & 3.48 & 3.74 \\
\hline & $R$ & 0.39 & 0.18 & 0.59 \\
\hline & \multicolumn{4}{|c|}{ Sequence of factors $\quad C>A>B$} \\
\hline & \multicolumn{4}{|c|}{ Optimal decision $C_{3} A_{1} B_{2}$} \\
\hline \multirow{6}{*}{$\begin{array}{c}\text { Specific energy } \\
w\end{array}$} & $K_{1}$ & 157.41 & 153.31 & 142 \\
\hline & $K_{2}$ & 159.88 & 161.52 & 160.08 \\
\hline & $K_{3}$ & 154.33 & 156.79 & 169.54 \\
\hline & $R$ & 5.55 & 8.21 & 27.54 \\
\hline & \multicolumn{4}{|c|}{ Sequence of factors $\quad C>B>A$} \\
\hline & \multicolumn{4}{|c|}{ Optimal decision $C_{1} B_{1} A_{3}$} \\
\hline
\end{tabular}
the same for the following tables.
Table 3 Results of range analysis

Table 4 Results of variance analysis

\begin{tabular}{ccccccc}
\hline Text Index & Source & $\begin{array}{c}\text { Sum of } \\
\text { squares }\end{array}$ & $\begin{array}{c}\text { Degree of } \\
\text { freedom }\end{array}$ & $\begin{array}{c}\text { Mean } \\
\text { squares }\end{array}$ & $F$ value & $P$ value \\
\hline $\begin{array}{c}\text { Forming } \\
\text { rate } \\
\lambda\end{array}$ & $A$ & 42.4939 & 2 & 21.2469 & 99.5899 & $0.0099^{* *}$ \\
& $C$ & 17.1307 & 2 & 8.5653 & 40.1480 & $0.0243^{*}$ \\
& Error & 0.4267 & 2 & 0.2133 & & \\
$\begin{array}{c}\text { Relax } \\
\text { density }\end{array}$ & $A$ & 0.0260 & 2 & 0.0130 & 167.2857 & $0.0059^{* *}$ \\
$\rho$ & $C$ & 0.0058 & 2 & 0.0029 & 37.0000 & $0.0263^{*}$ \\
& Error & 0.0002 & 2 & 0.0001 & & $0.0063^{* *}$ \\
\hline $\begin{array}{c}\text { Specific } \\
\text { energy }\end{array}$ & $B$ & 5.1544 & 2 & 2.5772 & 14.2589 & 0.0655 \\
$w$ & $C$ & 11.3208 & 2 & 5.6604 & 31.3172 & $0.0309^{*}$ \\
& Error & 0.3615 & 2 & 0.1807 & & $0.0028^{* *}$ \\
\hline
\end{tabular}

Note: $p \leq 0.01$ (Extremely significant), $0.01<p \leq 0.05$ (Significant).

Table 4 indicates that each factor has a different influence on the three indexes. For the molding rate index, the material granularity and length-to-diameter ratio have extremely significant influence on the test results $(p \leq 0.01)$, and the moisture content has significant influence $(0.01<p \leq 0.05)$. For the index of relax density, the particle size and length-to-diameter ratio of materials have a very significant effect on the test results $(p \leq 0.01)$, and the moisture content has a significant effect $(0.01<p \leq 0.05)$. For the specific energy consumption index, the length-to-diameter ratio has a very significant effect on the test results $(p \leq 0.01)$, the moisture content has a significant effect $(0.01<p \leq 0.05)$, and the material particle size has no significant effect $(p>0.05)$.

3.5.2 Comprehensive optimization of various factors

To obtain the ideal particle forming effect, it is necessary to have the molding rate and relax density as high as possible, while the specific energy consumption of forming may be as low as possible. The experimental analysis shows that the primary and secondary action sequence and significance of the three factors of material particle size, moisture content, and length-to-diameter ratio are different, and their optimal parameter combinations are also different. To further analyze the comprehensive influence of 
various factors on the particle forming index, the fuzzy comprehensive evaluation (FCE) method $^{[25]}$ was used to conduct a comprehensive optimization analysis, and the parameter combinations that made the three forming indexes as optimal as possible were selected. First, according to the importance of each index, the weights of molding rate, relaxation density, and specific energy consumption were determined to be $0.45,0.25$, and 0.30 , respectively, thus forming a weight distribution set $W=\left[\begin{array}{ll}0.45 & 0.25\end{array}\right.$ 0.30]. Then, the fuzzy comprehensive evaluation method was used to establish three index membership degree models, and dimensionless membership degree values with the same order of magnitude were obtained. The larger the index values of molding rate and relaxation density, the better. The membership model is shown in Equation (8). It is better for a smaller index value of molding energy consumption. The membership model is shown in Equation (9).

$$
\begin{gathered}
Q_{\text {in }}=\frac{R_{\text {imin }}-R_{\text {in }}}{R_{\text {imin }}-R_{\text {imax }}} \quad(n=1,2,3, \cdots, 9) \\
Q_{\text {in }}^{\prime}=\frac{R_{\text {imax }}-R_{\text {in }}}{R_{\text {imax }}-R_{\text {imin }}} \quad(n=1,2,3, \cdots, 9)
\end{gathered}
$$

where, $Q_{i n}$ is the membership value of index $R_{i}$ measured in $n$-th test when the index has a large value; $Q^{\prime}$ in is the membership value of index $R_{i}$ measured in the nth test when the index has a small value; $R_{\text {imax }}$ is the maximum value of index $R_{i} ; R_{\text {imin }}$ is the minimum value of index $R_{i} ; R_{i n}$ is the value of index $R_{i}$ of $n$-th test. According to Equations (8) and (9), the membership values of particle molding rate, relax density, and specific energy consumption can be calculated, whose values are $R_{1 n}, R_{2 n}$, and $R_{3 n}$, respectively, as shown in Table 4. The fuzzy relationship matrix $R$ composed of the membership values of three indicators is

$$
R=\left(\begin{array}{cccc}
r_{11} & r_{12} & \cdots & r_{1 m} \\
r_{21} & r_{22} & \cdots & r_{2 m} \\
\vdots & \vdots & \ddots & \vdots \\
r_{n 1} & r_{n 2} & \cdots & r_{n m}
\end{array}\right)
$$

The fuzzy comprehensive evaluation value set $U$ was obtained by the fuzzy transformation of fuzzy matrix $R$ and weight allocation set $W$, where $U=W \cdot R$, and the comprehensive scoring value of each experimental scheme was thus obtained, as shown in $U_{x}$ column in Table 5. The higher the scoring value, the better the particle forming quality of the scheme and the lower the specific energy consumption of the pellet.

Table 5 Comprehensive score

\begin{tabular}{ccccc}
\hline $\begin{array}{c}\text { Test } \\
\text { No. }\end{array}$ & $\begin{array}{c}\text { Membership } \\
\text { values of } \\
\text { molding rate } Q_{1 n}\end{array}$ & $\begin{array}{c}\text { Membership } \\
\text { values of relax } \\
\text { density } Q_{2 n}\end{array}$ & $\begin{array}{c}\text { Membership } \\
\text { values of specific } \\
\text { energy } Q_{3 n}^{\prime}\end{array}$ & Score $U_{x}$ \\
\hline 1 & 0.7649 & 0.7419 & 1.0000 & 0.8297 \\
2 & 0.1667 & 0.2258 & 0.2747 & 0.2139 \\
3 & 0 & 0 & 0.1617 & 0.0485 \\
4 & 0.8383 & 0.5806 & 0.4356 & 0.6531 \\
5 & 0.1002 & 0.0645 & 0 & 0.0612 \\
6 & 1.0000 & 0.7742 & 0.8069 & 0.8856 \\
7 & 0.6647 & 0.5484 & 0.3226 & 0.5330 \\
8 & 0.9980 & 1.0000 & 0.8391 & 0.9508 \\
9 & 0.8313 & 0.6774 & 0.5165 & 0.6984 \\
\hline
\end{tabular}

Range analysis and variance analysis were performed on the above comprehensive scoring results; the results are shown in Tables 6 and 7, respectively.

Combined with Table 6 of comprehensive score range analysis and Figure 4 of the influence trend of factor level on comprehensive score, it can be seen that the primary and secondary action sequence that comprehensively affects the molding performance of particles is length-to-diameter ratio $C$, particle size $A$, and moisture content $B$. The optimal parameter combination is $C_{1} A_{3} B_{1}$, i.e., length-to-diameter ratio $4: 1$, material particle size 6$9 \mathrm{~mm}$, and moisture content $8 \%$. Table 7 (results of comprehensive score analysis of variance) shows that at a $95 \%$ confidence level, the overall impact of particle size, moisture content, and length-to-diameter ratio on the particle forming quality

\begin{tabular}{|c|c|c|c|c|c|}
\hline \multicolumn{2}{|l|}{ Item } & $A$ & \multicolumn{2}{|c|}{$B$} & $C$ \\
\hline \multicolumn{2}{|l|}{$K_{1}$} & 1.0921 & \multicolumn{2}{|c|}{2.0158} & 2.6661 \\
\hline \multicolumn{2}{|l|}{$K_{2}$} & 1.5999 & \multicolumn{2}{|c|}{1.2259} & 1.5654 \\
\hline \multicolumn{2}{|l|}{$K_{3}$} & 2.1822 & \multicolumn{2}{|c|}{1.6325} & 0.6427 \\
\hline \multicolumn{2}{|c|}{ Total range $R$} & 1.0901 & \multicolumn{2}{|c|}{0.7899} & 2.0234 \\
\hline \multicolumn{6}{|c|}{ Sequence of factors $C>A>B$} \\
\hline \multicolumn{6}{|c|}{ Optimal decision $C_{1} A_{3} B_{1}$} \\
\hline Table 7 & \multicolumn{5}{|c|}{ Comprehensive score analysis of variance table } \\
\hline Source & $\begin{array}{l}\text { Sum of } \\
\text { squares }\end{array}$ & $\begin{array}{l}\text { Degree of } \\
\text { freedom }\end{array}$ & $\begin{array}{l}\text { Mean } \\
\text { squares }\end{array}$ & $F$ value & $P$ value \\
\hline$A$ & 0.1984 & 2 & 0.0992 & 285.8599 & 0.0035 \\
\hline$B$ & 0.1040 & 2 & 0.0520 & 149.8595 & 0.0066 \\
\hline$C$ & 0.6841 & 2 & 0.3421 & 985.7913 & 0.0010 \\
\hline Error & 0.0007 & 2 & 0.0003 & & \\
\hline
\end{tabular}
and energy consumption is extremely significant $(p \leq 0.01)$.

Table 6 Table of comprehensive rating range analysis

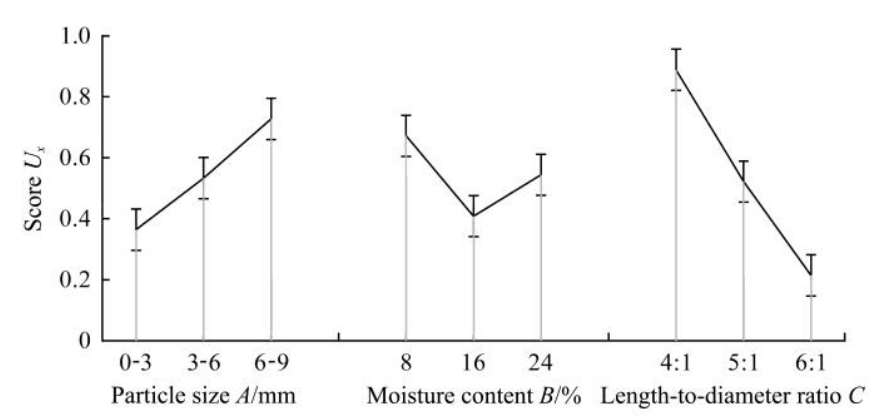

Figure 4 Influence trend chart of the factor level on comprehensive score

\subsection{Test verification}

The orthogonal test scheme does not include the optimal combination of parameters after comprehensive optimization. Therefore, to ensure the accuracy of optimized results, the optimal combination of parameters (i.e., material particle size of 6-9 mm, moisture content of $16 \%$, and length-to-diameter ratio of 4:1) was selected to conduct the verification test. To eliminate random errors, the experiment was repeated three times, and the average value was taken as the experimental verification value. The experimental results show that the particle molding rate is $97.80 \%$, relax density is $1.32 \mathrm{~g} / \mathrm{cm}$, and specific energy consumption is $44.17 \mathrm{~J} / \mathrm{g}$. Compared with the results of other orthogonal test schemes, the comprehensive performance of optimized granulation is better than that of other parameter combinations.

\section{Discussion}

(1) The length-to-diameter ratio has significant influence on molding rate, relax density, and specific energy consumption, mainly due to the length-to-diameter ratio determining the residence time and extrusion force of materials in die holes. The 
larger length-to-diameter ratio will result in higher filling degree of material. The closer the contact, the greater the pressure generated inside the material. Under high pressure, cellulose present in the material softens, and the Van der Waals' force, electrostatic attraction, and liquid adhesion between particles play a role. This changes the original physical and chemical properties of the material, thus changing the curing and molding results ${ }^{[26]}$. However, larger length-to-diameter ratio will result in higher energy consumption of compression. Therefore, the relationship between molding granules and granulation energy consumption should be considered comprehensively.

(2) Moisture content has a significant effect on molding rate and relax density. It also has a significant effect on comparative energy consumption. This is mainly because water is a natural adhesive medium, and proper moisture content can increase Van der Waals' force and form hydrogen bonds. It can also promote the precipitation of pectin, sugar, and other viscous substances in materials and promote molding. However, when the moisture content is too low, the resistance is large because the material is too dry. Therefore, the particles cannot be fully extended, resulting in poor molding effect and high specific energy consumption. On the other hand, when the moisture content is too high, excessive water will be squeezed out during the molding, forming a "water barrier" among the particle layers ${ }^{[27]}$. This hinders direct contact between molecules will make the particle layers unable to fit closely and thus decreasing the molding rate and relax density.

(3) Material particle size has a significant influence on particle molding rate and relax density. This is mainly because under certain pressure, the smaller the material particle size, the more severe is the damage of the smooth cutting on the outer surface of pineapple rind residue material. The larger the interaction area between small particles, the stronger the adhesive force between particles. This is more conducive to particle solidification and forming. However, when the particle size is too large, more cutin remains on the rind residue, and the gap between materials is too large. This will decrease the bonding performance, and thus decrease the molding ability and relax density.

\section{Conclusions}

(1) To quantitatively analyze the effects of pineapple rind residue particle size, moisture content, and die hole length-to-diameter ratio on the granulating quality and energy consumption, a single-mode hole compression molding test device was designed and manufactured in this study. A force analysis was carried out within the die material. The relationship between compression molding material in extrusion and die structural parameters (die cut area, perimeter, die hole length) and the hole wall friction coefficient equations were derived.

(2) Considering the molding quality and molding energy consumption, the primary and secondary factors affecting the comprehensive performance of pineapple rind residue compression molding were determined to be the length-to-diameter ratio, particle size, and moisture content. The optimal parameter combination is material particle size of 6-9 $\mathrm{mm}$, moisture content of $16 \%$, and length-to-diameter ratio of $4: 1$. Under these conditions, the molding rate of particles is $97.80 \%$, the relax density is $1.32 \mathrm{~g} / \mathrm{cm}$, and the specific energy consumption is $44.17 \mathrm{~J} / \mathrm{g}$. This study provides a reference for the selection of technological parameters and the development of molding equipment for pineapple rind residue particle molding.

\section{Acknowledgements}

The research work was supported by the Special Funds for Scientific Research in Public Welfare Industries (Agriculture) "Comprehensive Utilization of By-products of Horticultural Crop Products Processing" (Grant No. 201503142). The authors would also like to thank graduate students Bin Tang and Zheng Yin for their help with the experiment.

\section{[References]}

[1] Hossain M F. World pineapple production: An overview. African Journal of Food, Agriculture, Nutrition and Development, 2016; 16(4): 11443-11456.

[2] Dahunsi S O. Liquefaction of pineapple rind: Pretreatment and process optimization. Energy, 2019; 185:1017-1031.

[3] Liu H Q, Jiang W L. Development of pineapple industry in the world Prospect of Agricultural Production, 2015; 3: 49-53. (in Chinese)

[4] Rodsamran P, Sothornvit R. Preparation and characterization of pectin fraction from pineapple rind as a natural plasticizer and material for biopolymer film. Food and Bioproducts Processing, 2019; 118: 198-206.

[5] Wang F, Gao Y L, Shi J W. Study on extraction, stability and antioxidant activity of flavonoids from pineapple rinds. Journal of Shanxi Agricultural Sciences, 2014; 42(7): 672-677. (in Chinese)

[6] Tang X, Sun Y Y, Pan D D, Dai Y J, Zheng L T. Enzymatic extraction of polyphenols from pineapple rind and stability evaluation of the extracted polyphenols. Journal of Nuclear Agricultural Sciences, 2018; 32(2): 335-343. (in Chinese)

[7] Jiang Y C, Lin L J, Gong X, Huang X B, Zeng Y, Li J H. Effect of physical modification treatment on physico-chemical properties of modified dietary fiber from pineapple pomace. Chinese Journal of Tropical Crops, 2019; 40(5): 973-979. (in Chinese)

[8] Yang M, Chi X J. Research progress of comprehensive utilization of pineapple rind residue in China. China Fruit \& Vegetable, 2019; 39(8): 48-51. (in Chinese)

[9] Zhang B, Tian K P, Li X W, Huang J C, Shen C, Wang J G, et al. Fruit and vegetable pomace particle formation research. Journal of Chinese Agriculture Mechanical, 2015; 36(6): 328-331. (in Chinese)

[10] Tian K P, Zhang B, Li X W, Huang J C, Shen C. Study on die hole structure design and simulation optimization of fruit and vegetable skin residue granular molding machine. Journal of Agricultural Science and Technology, 2018; 20(6): 62-68. (in Chinese)

[11] Liu K K, Liao P W, Gong J X, Wang R B, Zhang A M. Research and analysis of the key technologies and equipment with fuel utilization of cotton stalk. Journal of Chinese Agriculture Mechanization, 2018; 39(1): 78-83. (in Chinese)

[12] Thoreson C P, Webster K E, Darr M J, Kapler E J. Investigation of process variables in the densification of corn stover briquettes. Energies, 2014; 7(6): 4019-4032.

[13] Garcia-Maraver A, Rodriguez M L, Serrano-Bernardo F, Diaz L F, Zamorano M. Factors affecting the quality of pellets made from residual biomass of olive trees. Fuel Processing Technology, 2015; 129: 1-7.

[14] Yumak H, Ucar T, Seyidbekiroglu N. Briquetting soda weed (Salsola tragus) to be used as a rural fuel source. Biomass \& Bioenergy, 2010; 34(5): 630-636.

[15] Kaewwinud N, Khokhajaikiat P, Boonma A. Effect of biomass characteristics on durability of Cassava stalk residues pellets. Research in Agricultural Engineering, 2018; 64(1): 15-19.

[16] Shaw M D, Tabil L G. Compression, relaxation, and adhesion properties of selected biomass grinds. Agricultural Engineering International: CIGR Journal, 2007; 9: 3675-3681.

[17] Hu J J, Lei T Z, He X F, Li Z F, Liu J W, Wang Z W, et al. Experimental research on the compressing molding parameter under cold conditions for wheat straw pellet fuel. Acta Energiae Solaris Sinica, 2008; 29(2): 241-245. (in Chinese)

[18] Dong L, Gai C, Dong Y P. Numerical study on process and influencing factors of biomass hydraulic briquetting. T Chin Soc Agric Mach, 2011; 42(7): 139-143. (in Chinese)

[19] Zhang X, Cai Z S, Zhang D Z, Zhang Z. Process optimization for densification of water hyacinth pellets fuel. Transactions of the CSAE, 2016; 32(5): 239-244. (in Chinese) 
[20] Huang X P, Wan F X, Huang J L, Wu J F, Zhang K L. Parameter optimization of granulated alfalfa pelleting process based on extrusion simulation experiment. Transactions of the CSAE, 2011; 27(11): 354-358. (in Chinese)

[21] Zou L, Bai H T. Theoretical analysis of geometric parameters of die hole of feed bulking machine. Transactions of the CSAM, 2008; 39(6): 203-204. (in Chinese)

[22] Tu D Y, Li A X, He G S. Parameter optimization of rice straw cold press process. Journal of Agricultural Science and Technology, 2015; 17(3): 56-62. (in Chinese)

[23] SC/T 6012-2002. Ministry of Agriculture of the People's Republic of
China. Test Method of Flat Die Pellet Feed Press. Beijing, 2002.

[24] NY/T 1881. Ministry of Agriculture of the People's Republic of China Test Method of Biomass Solid Molding Fuel. Beijing, 2010.

[25] Yu Z Y, Hu Z C, Yang K, Peng B L, Wu Feng, Xie H X. Design and experiment of root cutting device in garlic combine harvesting. Transactions of the CSAE, 2016; 32(22): 77-85. (in Chinese)

[26] Wu Y Y, Dong Y P, Wu Y S. Research on micro-mechanism of biomass briquet. Acta Energiae Solaris Sinica, 2011; 2: 124-127. (in Chinese)

[27] Tu D Y, Li A X, Hu Y R, Xie W. Lab-scale experimental study on the compressing molding parameters under cold condition for rice straw pellet. Chinese Journal of Agrometeorology, 2015; 36(4): 446-453. (in Chinese) 